\title{
Long non-coding RNA BCYRN1 exerts an oncogenic role in colorectal cancer by regulating the miR-204-3p/KRAS axis
}

Liu Yang ${ }^{1}$, Yinan Zhang ${ }^{1}$, Jun Bao ${ }^{2^{*}}$ (1) and Ji-Feng Feng ${ }^{2^{*}}$

\begin{abstract}
Background: It has been well documented that long non-coding RNAs (IncRNAs) regulate numerous characteristics of cancer, including proliferation, migration, metastasis, apoptosis, and even metabolism. LncRNA BCYRN1 (BCYRN1) is a newly identified brain cytoplasmic IncRNA with 200 nucleotides that was discovered to be highly expressed in tumour tissues, including those of hepatocellular carcinoma, gastric cancer and lung cancer. However, the roles of BCYRN1 in colorectal cancer (CRC) remain obscure. This study was designed to reveal the role of BCYRN1 in the occurrence and progression of CRC.
\end{abstract}

Methods: RT-PCR was used to detect the expression level of BCYRN1 in tumour tissues and CRC cell lines. BCYRN1 was knocked down in CRC cells, and cell proliferation changes were evaluated by cell counting kit-8 (CCK-8), 5-ethynyl-2'-deoxyuridine (EdU), and Ki-67 and proliferating cell nuclear antigen (PCNA) expression assays. Cell migration and invasion changes were evaluated by wound healing, Transwell and invasion-related protein expression assays. Flow cytometry analysis was used to assess whether BCYRN1 regulates the apoptosis of CRC cells. The dual luciferase reporter gene detects the competitive binding of BCYRN1 to miR-204-3p. In vivo experiments were performed to evaluate the effect of BCYRN1 on tumour development. TargetScan analysis and dual luciferase reporter gene assays were applied to detect the target gene of miR-204-3p. Rescue experiments verified that BCYRN1 affects CRC by regulating the effect of miR-204-3p on KRAS.

Results: We found that compared with normal tissues and human intestinal epithelial cells (HIECs), CRC tumour tissues and cell lines had significantly increased BCYRN1 levels. We further determined that knockdown of BCYRN1 inhibited the proliferation, migration, and invasion and promoted the apoptosis of CRC cells. In addition, bioinformatics analysis and dual luciferase reporter assay showed that BCYRN1 served as a competitive endogenous RNA (ceRNA) to regulate the development of CRC through competitively binding to miR-204-3p. Further studies proved that overexpression of miR-204-3p reversed the effects of BCYRN1 on CRC. Next, TargetScan analysis and dual luciferase reporter assay indicated that KRAS is a target gene of miR-204-3p and is negatively regulated by miR-204-3p. A series of rescue experiments showed that BCYRN1 affected the occurrence and development of CRC by regulating the effects of miR-204-3p on KRAS. In addition, tumorigenesis experiments in a CRC mouse model confirmed that BCYRN1 downregulation effectively inhibited tumour growth.

Conclusions: Our findings suggest that BCYRN1 plays a carcinogenic role in CRC by regulating the miR-204-3p/KRAS axis.

\footnotetext{
*Correspondence: baojun70s@126.com; fengjifeng_jsch@sohu.com

${ }^{2}$ Department of Chemotherapy, The Affiliated Cancer Hospital of Nanjing

Medical University \& Jiangsu Cancer Hospital \& Jiangsu Institute of Cancer Research, No. 42 Baiziting, Nanjing, China

Full list of author information is available at the end of the article
}

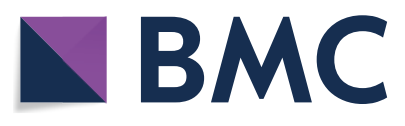

(c) The Author(s) 2020. This article is licensed under a Creative Commons Attribution 4.0 International License, which permits use, sharing, adaptation, distribution and reproduction in any medium or format, as long as you give appropriate credit to the original author(s) and the source, provide a link to the Creative Commons licence, and indicate if changes were made. The images or other third party material in this article are included in the article's Creative Commons licence, unless indicated otherwise in a credit line to the material. If material is not included in the article's Creative Commons licence and your intended use is not permitted by statutory regulation or exceeds the permitted use, you will need to obtain permission directly from the copyright holder. To view a copy of this licence, visit http://creativeco mmons.org/licenses/by/4.0/. The Creative Commons Public Domain Dedication waiver (http://creativecommons.org/publicdomain/ zero/1.0/) applies to the data made available in this article, unless otherwise stated in a credit line to the data. 
Keywords: IncRNA BCYRN1, miR-204-3p, KRAS, Colorectal cancer

\section{Background}

Colorectal cancer (CRC) has a high incidence and mortality worldwide; specifically, it ranks third in cancer morbidity and second in cancer mortality globally $[1,2]$. CRC has become one of the most common and dangerous malignancies in China [3]. The mainstay treatment for CRC is surgery, chemotherapy, radiotherapy and other comprehensive therapies, but the 5-year survival rate has not changed significantly in recent decades and is still poor $[4,5]$. Therefore, delivering high-efficiency CRC diagnosis and treatment methods should be the aim of all physicians in internal medicine research. The present study aims to provide an up-to-date molecular mechanism of CRC occurrence.

Recently, epigenetic changes have been reported to play important roles in the occurrence and progression of malignancies [6, 7]. LncRNAs are a class of transcripts with lengths greater than 200 nucleotides and have limited or non-protein coding potential [8-10]. Various studies have demonstrated that IncRNAs participate in the multilevel regulation of gene expression by targeting microRNAs (miRNAs), mRNAs, and even proteins $[11,12]$. Furthermore, growing evidence has proven that lncRNAs regulate multiple characteristics of cancer, such as proliferation, migration, apoptosis, and even metastasis $[13,14]$. BCYRN1 is a brain cytoplasmic lncRNA that is newly identified and activated by c-MYC [15]. It has been reported that BCYRN1 is highly expressed in various tumour tissues [16], such as hepatocellular carcinoma [17], gastric cancer [18], and lung cancer [15] but is expressed at lower levels in healthy control (HC) tissues. In addition, BCYRN1 plays an important role in inhibiting smooth muscle differentiation and vascular development in the cardiovascular system [19, 20]. However, little is known about the role of BCYRN1 in CRC, which aroused our great interest.

MiRNAs are a group of small, non-coding RNAs with nucleotides of approximately 22 and are identified as negative regulators [21, 22]. miRNAs can act as targets of lncRNAs and participate in the regulation of gene expression. Specifically, miRNA is involved in gene expression by binding to the complementary sequence location of the $3^{\prime}$-untranslated region (3'-UTR) of target mRNA to disturb the structural stability and translation of mRNAs $[23,24]$. Increasing evidence has demonstrated that miRNAs play crucial roles in cancer, cardiovascular disease and other diseases $[22,25]$ by regulating relative cell proliferation, migration, invasion and apoptosis [23]. On the other hand, lncRNAs competitively bind to miRNAs as ceRNAs, resulting in the inability of the miRNAs to combine with their corresponding mRNAs, thus regulating gene expression and function [11, 26, 27].

The present study aimed to determine the function and underlying mechanism of BCYRN1 in CRC progression. Our research determined that BCYRN1 plays an oncogenic role in CRC by regulating the miR-204-3p/KRAS axis, revealing a novel mechanism for the occurrence and development of CRC and providing a novel therapeutic and prognostic target for CRC.

\section{Materials and methods}

\section{Experimental human tissues and animals}

The research tissues of CRC patients and healthy controls were obtained from The Affiliated Cancer Hospital of Nanjing Medical University, and all patients signed written consent forms. Tissue debris was immediately frozen in liquid nitrogen after surgery and stored at $-80{ }^{\circ} \mathrm{C}$. All animal experiments were designed according to the standards of the Guide for the Care and Use of Laboratory Animals (NIH, 8th edition, 2011). The Ethics Committee of The Affiliated Cancer Hospital of Nanjing Medical University approved the present research.

\section{Cell culture}

Four human CRC cell lines, LoVo, HCT116, SW480, and SW620 and HIECs, were purchased from American Type Culture Collection (ATCC; Manassas, VA, USA) and cultured in DMEM (Gibco, Grand Island, NY, USA) containing 10\% foetal bovine serum (FBS; Gibco, Grand Island, NY, USA), $100 \mathrm{IU} / \mathrm{mL}$ penicillin and $1 \times 10^{5} \mu \mathrm{g} /$ $\mathrm{mL}$ streptomycin in a humidified atmosphere containing $5 \% \mathrm{CO}_{2}$ at $37^{\circ} \mathrm{C}$.

\section{Cell transfection}

shRNA-BCYRN1 (sh-BCYRN1), shRNA-KRAS (shKRAS) and corresponding shRNA negative control (shNC) transfection were performed using Lipofectamine 3000 reagent (Invitrogen, Carlsbad, CA, USA) according to the manufacturer's instructions. In addition, RNAifec$\operatorname{tin}^{\mathrm{TM}}$ transfection reagent, NC, miR-204-3p mimic and miR-204-3p inhibitor were obtained from Applied Biological Materials Inc. (Richmond, BC, Canada). HIECs and CRC cells were cultured in 6-well plates for transfection, and the efficiency of transfection was determined by qRT-PCR. 


\section{RNA extraction and qRT-PCR}

Total RNA was extracted from human tissues, mouse tissues and cells with TRIzol reagent (Life Technologies, Gaithersburg, MD, USA). Reverse transcription experiments were performed with PrimeScript ${ }^{\circledR}$ RT reagent Kits (Takara, Otsu, Shiga, Japan) and a StepOnePlus ${ }^{\text {TM }}$ Real-Time PCR System (Applied Biosystems, Foster City, CA, USA). Next, quantitative reactions were performed with SYBR Green RT-PCR (Takara Biotechnology Co., Ltd., Tokyo, Japan) using the StepOnePlus ${ }^{\text {TM }}$ system. GAPDH and U6 snRNA were used as the internal controls.

\section{Evaluation of cell proliferation}

Cell proliferation was detected by cell counting kit-8 (CCK-8) assay, 5-ethynyl-2'-deoxyuridine (EdU) incorporation test, and nucleoprotein Ki-67 and proliferating cell nuclear antigen (PCNA) expression analysis. First, for the CCK-8 experiment, cells were seeded into a 96-well plate with $10 \%$ FBS DMEM for $24 \mathrm{~h}$. Then, the medium was changed with serum-free DMEM for CCK-8 kit (Beyotime Biotechnology, Shanghai, China) detection. A microplate reader (Model ELX800, BioTek, Vermont, USA) was used to determine the absorbance at $450 \mathrm{~nm}$, which reflects cell viability and proliferation. Second, cells were seeded into a 24-well plate for EdU assessment. After $48 \mathrm{~h}$ of transfection, DNA synthesis was examined with an EdU incorporation assay (Guangzhou RiboBio, Guangzhou, China). The proportion of EdU-positive cells was analysed by fluorescence microscopy (DP70, Olympus Optical, Tokyo, Japan). Finally, cell proliferation was evaluated by Ki-67 and PCNA expression. Ki-67 is a nucleoprotein that is a marker of tumour proliferation [28]. PCNA acts on chromatin and participates in all aspects of the DNA replication chain [25]. The expression levels of Ki-67 and PCNA can be used to evaluate the status of cell proliferation.

\section{Evaluation of cell migration and invasion}

Cell migration and invasion were assessed with the wound healing assay, Transwell assay and invasionrelated protein expression. For the wound healing assay, cells were seeded into a 6-well plate. A sterile 1-mL pipette tip was used to scratch the bottom of the plate to form a gap. The images of cell migration were captured at $0 \mathrm{~h}$ and $48 \mathrm{~h}$ after transfection, respectively, with an inverted microscope (Axio Vert. A1, Zeiss, Oberkochen, Germany). The scratch distance at $48 \mathrm{~h}$ was subtracted from the scratch distance at $0 \mathrm{~h}$ to calculate the percentage closure of the wound [29]. The average distance of migration reflects the cell migration capacity. For the Transwell assay, cells were plated into the no FBS medium in the upper chamber of a 12-well Transwell with an
8- $\mu \mathrm{m}$ pore size (Merck $\mathrm{kGaA}$, Darmstadt, Germany). FBS medium (10\%) was added to the lower chamber. After $24 \mathrm{~h}$, the cells that migrated to the submembrane surface were stained with crystal violet. Stained cells were counted in 6 randomly selected regions.

\section{Flow cytometric analysis}

Flow cytometric analysis was designed to evaluate whether BCYRN1 regulated the apoptosis of CRC cells. Consistently, cells were seeded into a 6-well plate. The cells were collected and stained with annexin V-FITC (Beyotime Biotechnology, Shanghai, China) and propidium iodide (PI) reagent after $48 \mathrm{~h}$ transfection according to the manufacturer's instructions. Then, cell apoptosis was determined by flow cytometry (BD FACSCalibur cytometer, Becton Dickinson, San Jose, CA, USA).

\section{Dual luciferase reporter assay}

The partial sequences of BCYRN1 and the $3^{\prime}$-untranslated region (3'-UTR) of KRAS containing the putative binding sites of miR-204-3p were synthesized and inserted into a luciferase reporter vector plasmid (GenePharma, Shanghai, China). According to the instructions, firefly luciferase reporter plasmids and equal amounts of miR-204-3p mimics or NC mimics were transfected into cells. Then, the relative luciferase activities were detected by the Dual-Luciferase Reporter Assay System (Promega, Madison, WI, USA) on a Luminometer 20/20n (Turmer Biosystems, Sunnyvale, CA, USA) after transfection for $48 \mathrm{~h}$. Renilla luciferase activity was employed as an internal control for cellular density and transfection efficiency.

\section{In vivo experiments}

The nude mice were injected subcutaneously with CRC cells (approximately $10^{6}$ cells) into the right flanks. Then, sh-NC or sh-BCYRN1 was directly injected into the mice to knockdown BCYRN1. Tumour size was measured every 5 days for a total of 6 measurements. At the end of the experiments, mice were humanely sacrificed with overdose anaesthesia for the collection of tumours. Each tumour was weighed to evaluate the effects of BCYRN1 on the development of tumours.

\section{Immunohistochemistry}

Tumours were obtained, fixed in $4 \%$ paraformaldehyde, embedded in paraffin, and then cut into $4 \mu \mathrm{m}$ sections. After deparaffinization and blocking, the sections were incubated with primary anti-Ki-67 (1:100; Abcam; Cambridge, MA, USA) overnight at $4{ }^{\circ} \mathrm{C}$. The immunofluorescence images were captured with an Olympus BX51 microscope (Olympus, Tokyo, Japan) after incubating horseradish peroxidase-conjugated goat anti-rabbit antibody. 


\section{HE staining and TUNEL staining}

Haematoxylin-eosin (HE) staining and terminal deoxynucleotidyl transferase (TdT)-mediated dUTP nick-end labelling (TUNEL) staining were performed to detect cancer cell apoptosis in mouse tumour tissues. Briefly, mouse tumour tissues were prefixed, and the paraffinembedded sections were stained with haematoxylineosin and TUNEL (containing $2 \mu \mathrm{L}$ TdT enzyme, $48 \mu \mathrm{L}$ Fluorescent labelling solution and $50 \mu \mathrm{L}$ TUNEL detection solution) according to the manufacturer's instructions. The images were obtained with an Olympus BX51 microscope (Olympus, Tokyo, Japan) coupled with an Olympus DP70 digital camera.

\section{Western blot analysis}

Cyclooxygenase-2 (Cox-2) is an enzyme complex that plays significant roles in metastasis and invasion of malignancies [30]. The matrix metalloproteinase (MMP) family, especially the gelatinases MMP-2 and MMP-9, are recognized as markers of tumour migration and invasion [31]. The expression of Cox-2, MMP-2 and MMP-9 can reflect the status of cell migration and invasion. The protein expression levels of Ki-67, PCNA, Cox-2, MMP-2, MMP-9, Bax, Bcl-2, cleaved caspase-3, cleaved caspase-9 and KRAS were detected by Western blot analysis. Total protein was extracted, and equal amounts of protein $(30 \mu \mathrm{g})$ were separated by SDS-PAGE and then transferred to PVDF membranes. Protein bands were visualized with the Enhanced Chemiluminescence Detection Kit (Thermo Fisher Scientific, Rockford, IL, USA). All primary antibodies were obtained from Abcam (Cambridge, MA, USA). The dilution of PCNA antibody was 1:5,000, and the dilution ratio of other primary antibodies was $1: 1,000$.

\section{Statistical analysis}

Experiments were conducted in a randomized, doubleblinded situation. Data are expressed as the mean $\pm S D$. Student's unpaired t-test was used to compare the differences between two groups. One-way or two-way ANOVA followed by post hoc Bonferroni test was employed for multiple comparisons. Statistical significance was considered as ${ }^{*} P<0.05,{ }^{* *} P<0.01$, and ${ }^{* * * *} P<0.001$.

\section{Results}

BCYRN1 is highly expressed in the tissues and cell lines of CRC

In our preliminary study, we noted that BCYRN1 was highly expressed in CRC tissues (Fig. 1a). Then, we examined the expression levels of BCYRN1 in HIECs and four types of human CRC cell lines. Consistently, BCYRN1 expression was significantly increased in CRC cell lines (Fig. 1b). These data aroused our interest, as they suggested that BCYRN1 levels may be closely related to the development of CRC.

\section{Knockdown of BCYRN1 inhibits the proliferation and promotes the apoptosis of CRC cells}

To further illuminate the roles of BCYRN1 in CRC, a series of experiments were designed in LoVo and SW480 cells. First, we used sh-BCYRN1 to downregulate endogenous BCYRN1, and the knockdown efficiency is shown in Fig. 2a. CCK-8 and EdU assays showed that downregulation of BCYRN1 evidently decreased the proliferation of LoVo and SW480 cells (Fig. 2b, c). Furthermore, knockdown of BCYRN1 obviously inhibited the expression of proliferation-related proteins, such as PCNA and Ki-67 (Fig. 2d). Subsequently, we detected cell apoptosis by flow cytometry analysis. The results showed that knockdown of BCYRN1 significantly increased apoptosis of LoVo and
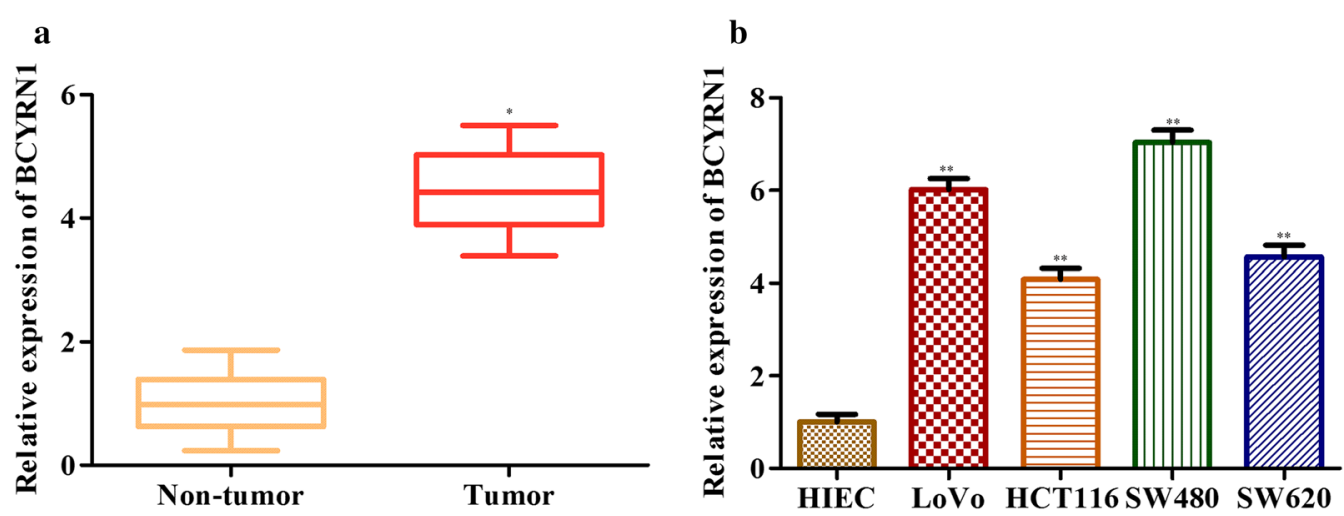

Fig. 1 LnCRNA BCYRN1 is highly expressed in the tissues and cell lines of CRC. a BCYRN1 levels in HC tissues and CRC tumour tissues. Data from 15 HC people and 15 GC patients. b BCYRN1 levels in HIECs and CRC cell lines. Values are the mean \pm SD. ${ }^{*} P<0.05,{ }^{* *} P<0.01$, and ${ }^{* *} P<0.001$ vs. HCs or HIECs. $n=3$ per group 
SW480 cells (Fig. 2e). In addition, several pro-apoptosis proteins, including Bax, cleaved caspase- 3 and cleaved caspase-9 [32, 33], were highly expressed in LoVo and SW480 cells when they were treated with sh-BCYRN1. In contrast, the expression of $\mathrm{Bcl}-2$, an inhibitor of cell apoptosis [33], was abolished in the sh-BCYRN1 group (Fig. 2f). These results indicated that BCYRN1 knockdown reduced proliferation while promoting the apoptosis of CRC cells.

\section{Downregulation of BCYRN1 decreases the migration and invasion of CRC cells}

Next, we investigated the effects of BCYRN1 expression on CRC cell migration and invasion. Both wound healing and Transwell assays revealed that the migration and invasion of LoVo and SW480 cells were effectively suppressed by sh-BCYRN1 transfection (Fig. 3a, b). Consistently, migration- and invasion-related proteins, such as Cox-2, MMP-2 and MMP-9, were inhibited in LoVo and SW480 cells treated with sh-BCYRN1 (Fig. 3c). These results elucidated that $\mathrm{BCYRN1}$ levels were closely related to the migration and invasion properties of CRC cells.

\section{miR-204-3p is a target gene of BCYRN1}

A recent report showed that lncRNAs function as ceRNAs to competitively bind to miRNAs [11]. To illuminate the potential regulatory mechanism through which BCYRN1 affects CRC progression, we performed bioinformatic analysis with LncBase Predicted V.2 and found that BCYRN1 possesses a putative binding site for miR204-3p (Fig. 4a). We further retrieved potential targets of BCYRN1 in different databases. To determine the interaction of BCYRN1 and miR-204-3p, we performed dual luciferase reporter analysis and found a significant reduction in the luciferase activity of BCYRN1 WT when LoVo or SW480 cells were transfected with miR-204-3p mimics. However, there was no notable difference in the luciferase activity of BCYRN1 Mut between the miR-204-3p mimic group and the negative control (NC) group (Fig. 4b). Accordingly, downregulation of BCYRN1 distinctly increased the expression of miR-204-3p in LoVo and SW480 cells (Fig. 4c). In addition, qRT-PCR results revealed that miR-204-3p was significantly reduced in CRC tumour tissues and cell lines, especially in LoVo and SW480 cells (Fig. 4d, e).
These data proved that miR-204-3p is a target gene of BCYRN1 and is negatively regulated by BCYRN1.

\section{Effects of miR-204-3p on CRC cell proliferation, migration, invasion and apoptosis}

We then studied the role of miR-204-3p in the regulation of CRC progression. First, we used miR-204-3p mimics to treat LoVo and SW480 cells. The overexpression efficiency of miR-204-3p is shown in Fig. 5a. Then, we found that miR-204-3p upregulation apparently inhibited cell proliferation, as demonstrated by CCK-8 and EdU assays (Fig. 5b, c). In addition, flow cytometry analysis revealed that miR-204-3p overexpression accelerated cell apoptosis (Fig. 5d). Consistently, CRC cell migration was obviously decreased by miR-204-3p mimics (Fig. 5e). These results concluded that miR-204-3p upregulation inhibits CRC cell proliferation, migration and invasion while promoting CRC cell apoptosis.

\section{KRAS is a target gene of miR-204-3p}

We further explored the underlying mechanism of miR204-3p in CRC. TargetScan, a miRNA target prediction database, was used to predict the downstream gene of miR-204-3p. We found that KRAS was a possible target gene of miR-204-3p, and the predicted position of the combination was in the 310-317 region of the KRAS $3^{\prime}$-UTR (Fig. 6a). It is generally known that KRAS is a crucial carcinogenic factor [34]. Moreover, the results of dual luciferase reporter analysis confirmed that KRAS was indeed the target gene of miR-204-3p (Fig. 6b). More significantly, overexpression of miR-204-3p inhibited the expression of KRAS mRNA and protein in LoVo and SW480 cells (Fig. 6c, d). We next investigated the relationship between KRAS levels and CRC progression. We noted that KRAS was highly expressed in CRC tumour tissues and cell lines (Fig. 6e, f). These results indicated that KRAS is a target gene of miR-204-3p and is negatively regulated by miR-204-3p.

\section{Effects of the BCYRN1/miR-204-3p/KRAS axis on CRC cell proliferation, migration, invasion and apoptosis}

To understand the effects of the BCYRN1/miR-204-3p/ KRAS axis on CRC progression, we designed a series

(See figure on next page.)

Fig. 2 The effects of BCYRN1 knockdown on CRC cell proliferation and apoptosis. a BCYRN1 levels in LoVo and SW480 cells. b CRC cell proliferation was detected with CCK-8 assay. $\mathbf{c}$ The fluorescence images and data statistics chart of the EdU assay. d The expression of PCNA and Ki-67. e Original images and histogram of the flow cytometry assay. $\mathbf{f}$ The expression of apoptosis-related proteins in LoVo cells and SW480 cells. Values are the mean \pm SD. ${ }^{*} P<0.05,{ }^{* *} P<0.01$, and ${ }^{* *} P<0.001$ vs. sh-NC. $n=3$ per group 

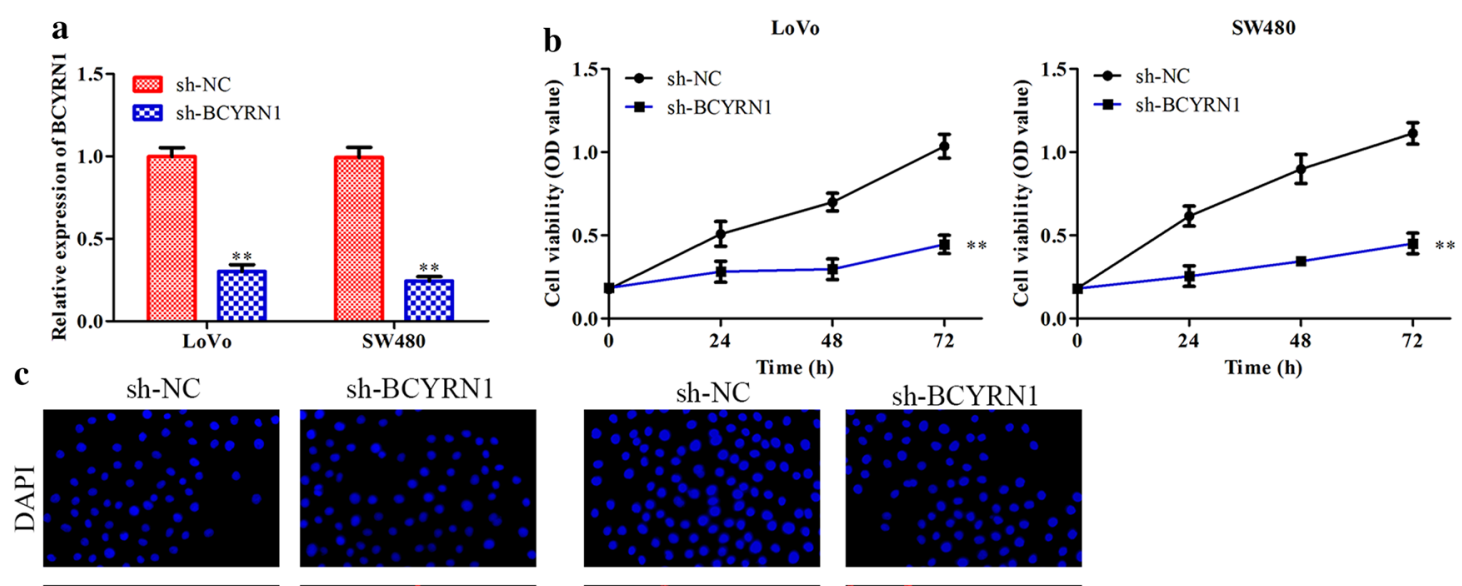

sh-BCYRN1
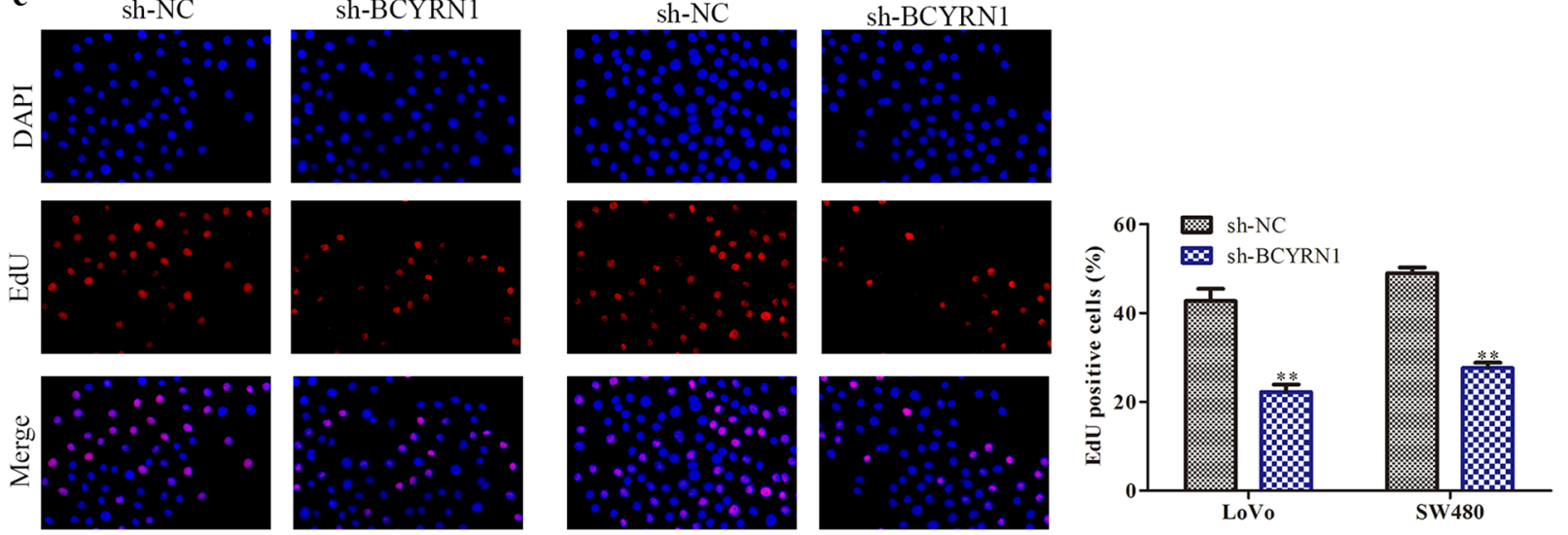

d
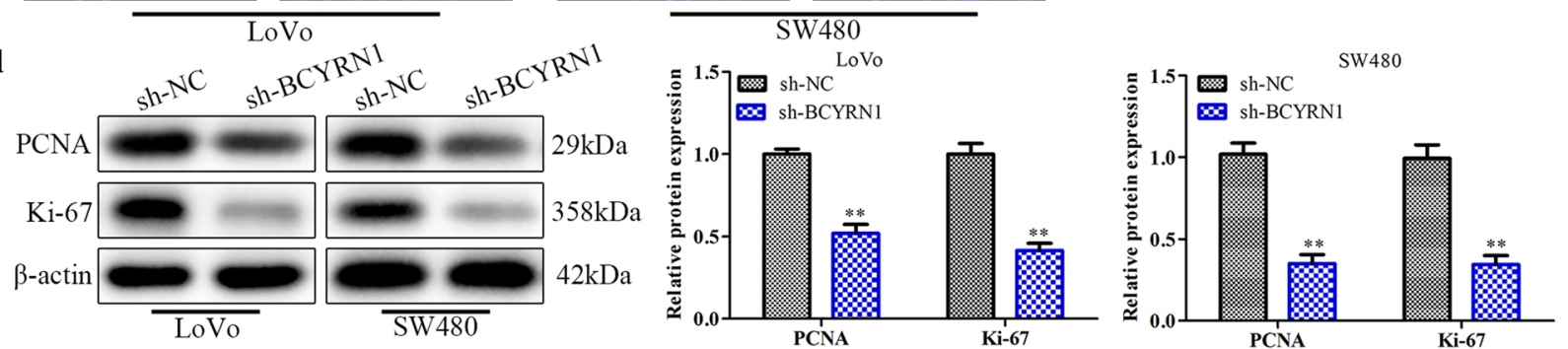

e
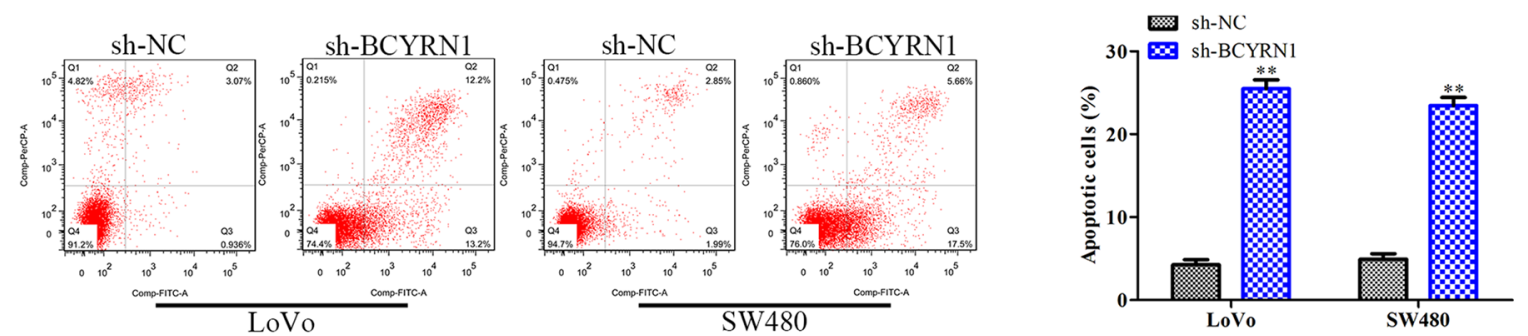

f
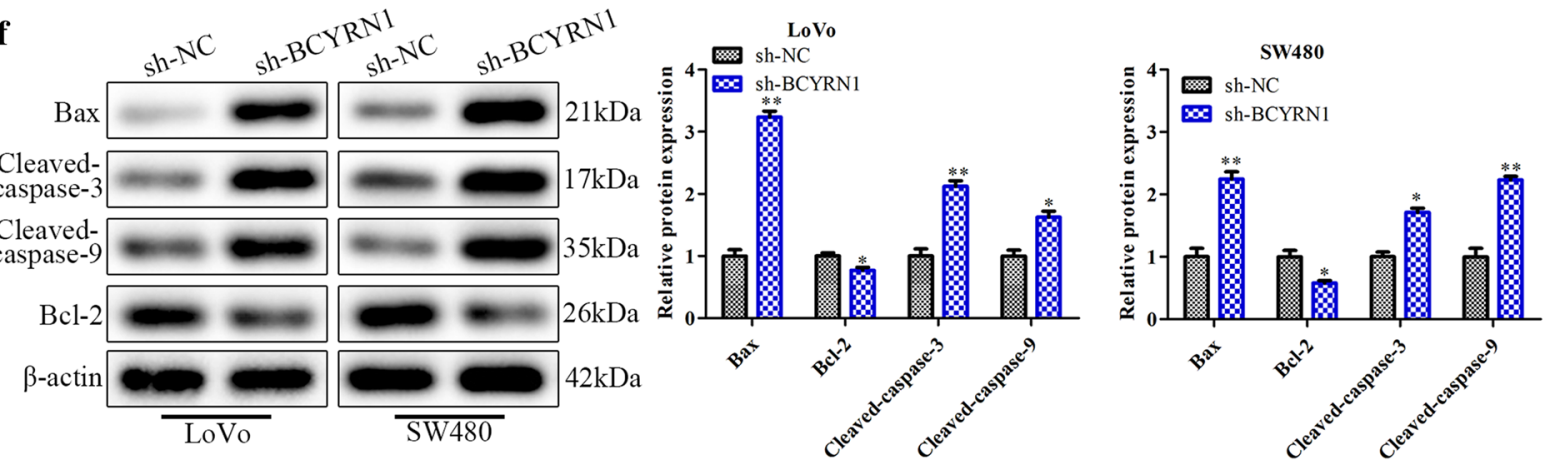


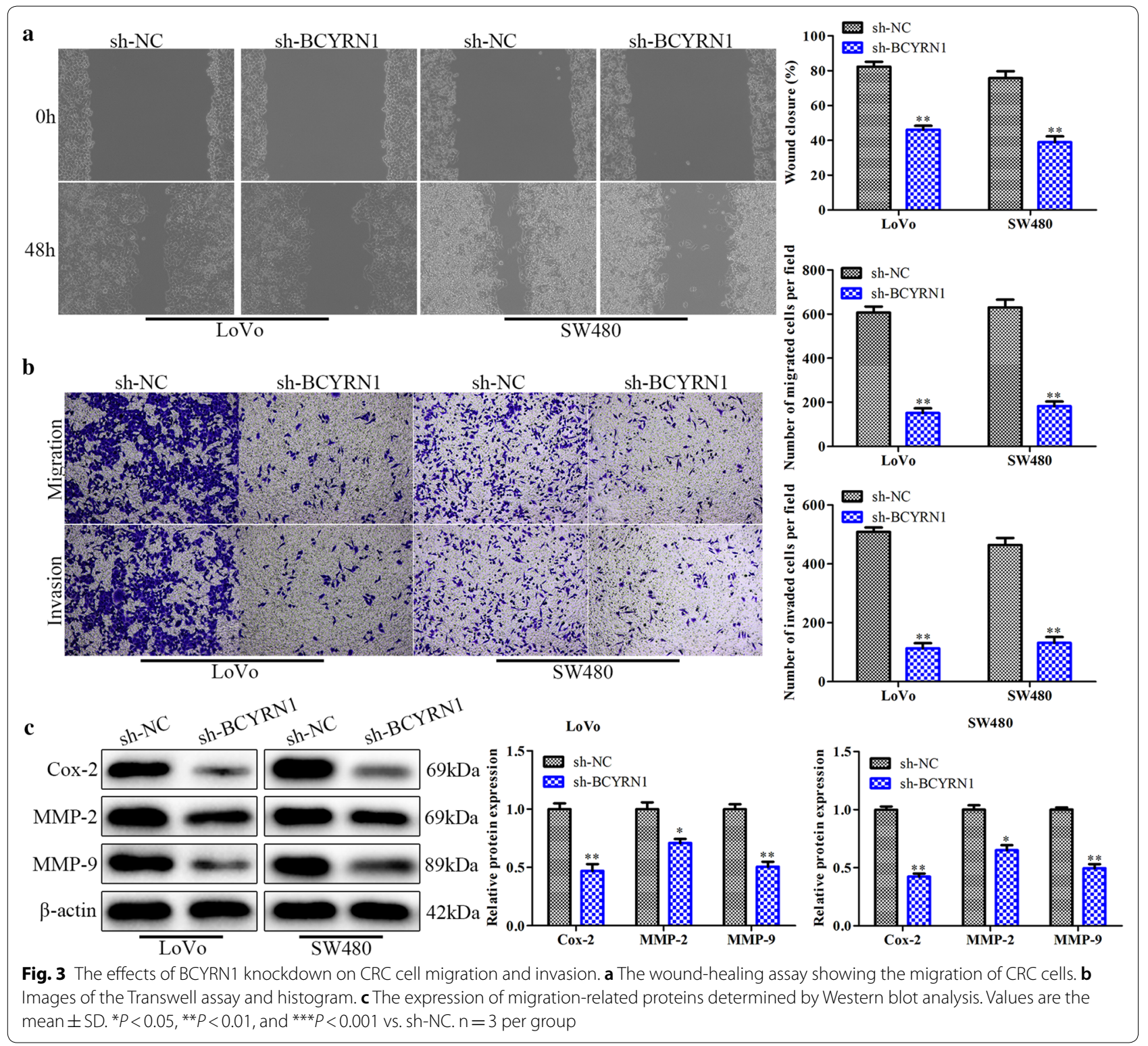

of rescue experiments using miR-204-3p inhibitor, shKRAS and sh-BCYRN1 in LoVo and SW480 cells. The knockdown efficiency of miR-204-3p and KRAS in CRC cells treated with sh-BCYRN1 is shown in Fig. 7a. Then, we found that the miR-204-3p inhibitor significantly facilitated the proliferation of LoVo and SW480 cells treated with sh-BCYRN1, whereas sh-KRAS effectively reversed these effects (Fig. $7 \mathrm{~b}, \mathrm{c}$ ). Furthermore, the miR-204-3p inhibitor abolished the apoptosis of shBCYRN1-treated CRC cells, whereas sh-KRAS relieved these phenomena (Fig. 7d). In addition, the migration of sh-BCYRN1-treated CRC cells was evidently increased in the miR-204-3p inhibitor-treated group, while sh-KRAS reversed these roles (Fig. 7e). These data indicated that BCYRN1 worsens CRC progression at least in part by inhibiting miR-204-3p levels and promoting KRAS expression.

\section{Knockdown of BCYRN1 inhibits tumour growth}

To evaluate the therapeutic potential of BCYRN1 in CRC patients, we performed BCYRN1 knockdown by using sh-BCYRN1 in CRC model mice. Encouragingly, knockdown of BCYRN1 significantly decreased the tumour volume and weight of CRC model mice (Fig. 8a-c). HE staining, immunohistochemical staining and TUNEL staining showed that downregulation of BCYRN1 inhibited Ki-67 expression and promoted cancer cell apoptosis (Fig. 8d). More importantly, sh-BCYRN1 treatment 


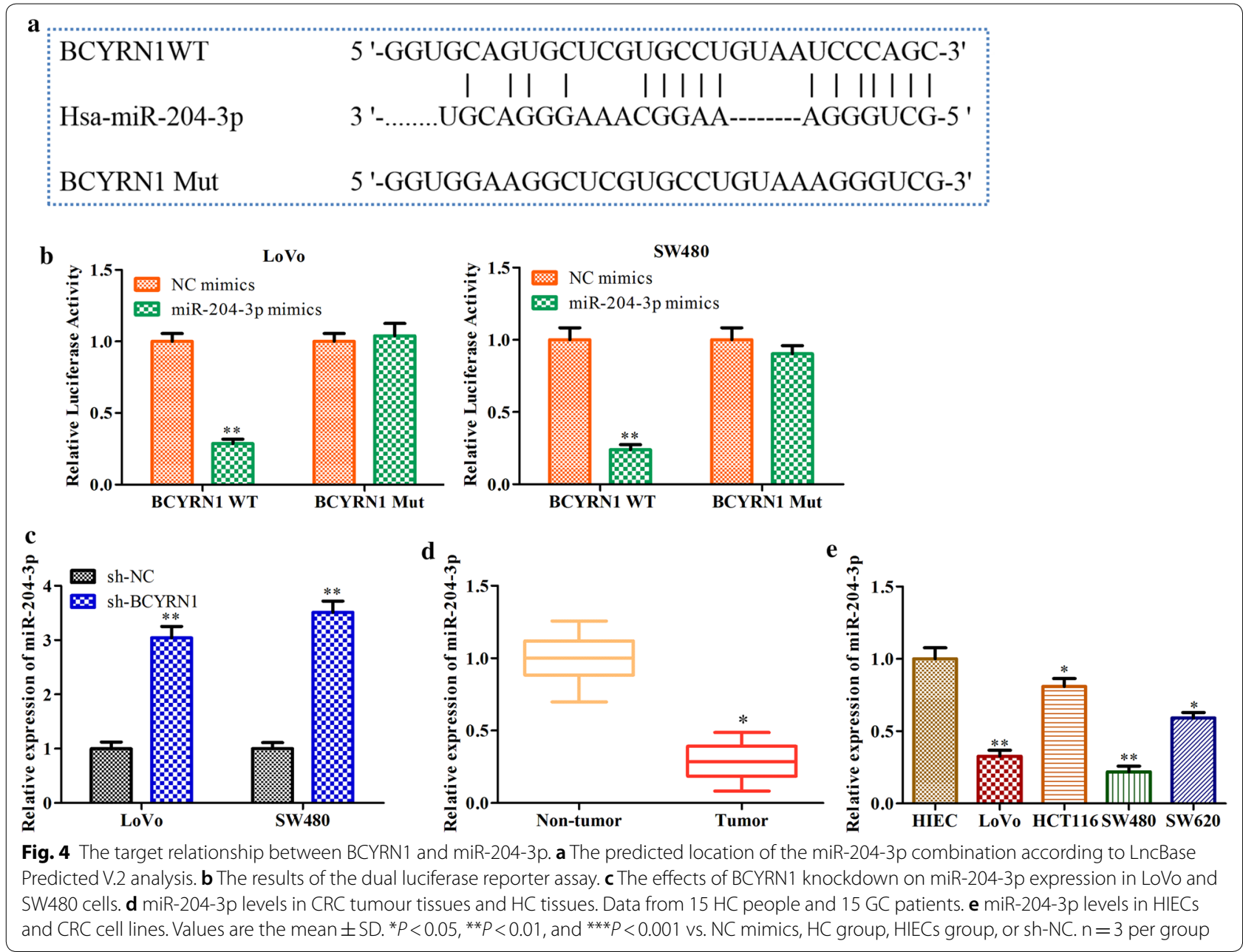

obviously promoted the expression of miR-204-3p and decreased the expression of KRAS (Fig. 8e). These results revealed that knockdown of BCYRN1 inhibited the development of CRC.

\section{Discussion}

Cancer mortality remains an enormous public health challenge on a global scale [35]. Although new advances have been made in the genomic changes in most different cancers [36], the molecular mechanisms underlying the development of CRC are poorly understood. Moreover, compared with patients with other malignancies, a large number of CRC patients have distant metastasis at the early stage of diagnosis, which leads to a low 5-year survival rate of approximately $10 \%-20 \%$ [37]. In addition, therapeutic methods are very limited in advanced CRC patients and mainly involve combinations of toxic drugs, such as 5-fluorouracil, irinotecan and/or oxaliplatin, for chemotherapy. Therefore, the molecular mechanism involved in the occurrence and development of
CRC needs to be explored urgently. Our current study revealed an important new mechanism of the occurrence of CRC and provided a novel idea for the effective treatment of CRC, briefly, to control CRC progression by blocking BCYRN1.

LncRNAs have been shown to play vital roles in the regulation of cancer progression [13, 14], and the main functions of lncRNAs are recruiting chromatin modification complexes for transcriptional regulation [38] and interacting with miRNAs, mRNAs, and/or proteins for posttranscriptional regulation [11, 12]. It has been proven that one of most common and crucial roles of lncRNA is their functions as a "miRNA sponges" [27, 39]. This means that IncRNA can be used as a bait to bind with a specific miRNA, resulting in the inability of the miRNA to combine with its corresponding mRNA. BCYRN1 is a newly identified lncRNA with similar characteristics [15]. Previous studies have revealed that miRNAs, such as miR-125-5p, miR-149 and miR-490-3p, act as targets of BCYRN1 in hepatocellular carcinoma, 


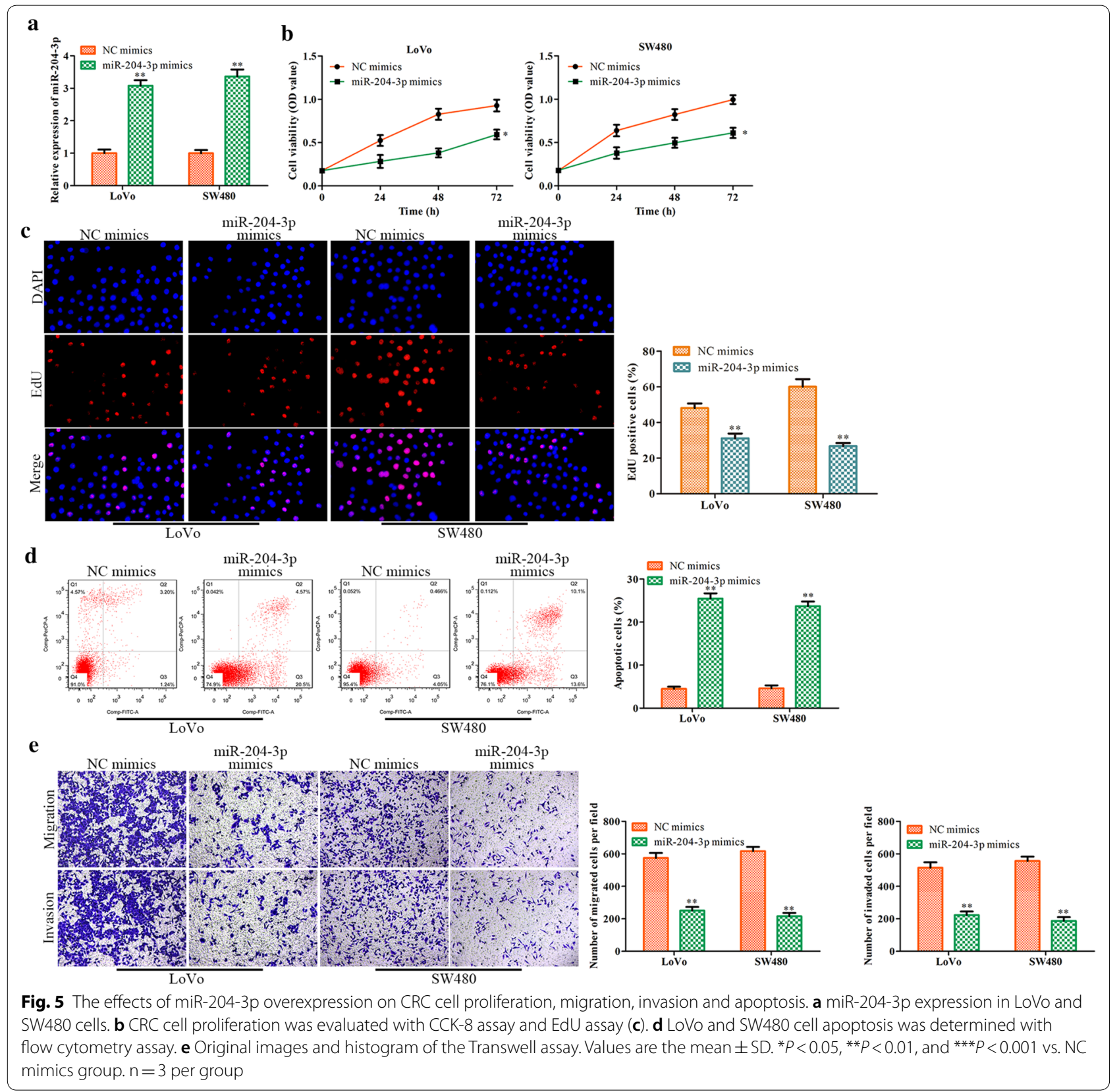

human glioma and lung cancer, respectively $[17,40,41]$. Recently, the relationship between BCYRN1 and CRC has been gradually observed. Gu et al. first found that BCYRN1 levels were closely related to the occurrence, development and prognosis of CRC, and microarray bioinformatics analysis confirmed that the possible target of BCYRN1 was the NRP3 gene [42]. Moreover, Yu $\mathrm{JH}$ and Chen Y proved that BCYRN1 levels were significantly increased in tumour tissues and cell lines (SW620) of CRC by a series of well-designed in vitro experiments [43]. However, it remains obscure whether BCYRN1 can control miRNAs to regulate the progression of CRC. Our present study indicated that BCYRN1 is involved in the occurrence and development of CRC through specific binding to a new target miRNA: miR-204-3p. We found that BCYRN1 was highly expressed, while the expression levels of miR-204-3p were significantly reduced in the tissues and cell lines of CRC compared to those of controls. In addition, knockdown of BCYRN1 distinctly increased miR-204-3p expression. These findings provide new insights into the mechanism of $\mathrm{CRC}$ progression and the treatment of CRC. 
a

\begin{tabular}{|c|c|}
\hline KRASWT & $\begin{array}{l}5^{1}-\ldots \text { CUCUAAGUGCCAGUAUUCCCAGA...-3' } \\
|||||| \mid\end{array}$ \\
\hline Hsa-miR-204-3p & $3^{\prime}-\ldots . . . .$. UGCAGGGAAACGGAAGGGUCG-5 ' \\
\hline KRAS Mut & $5^{\prime}-\ldots$ CUCUAAGUGCCAGUAAAGGGUCA $\ldots-.3{ }^{\prime}$ \\
\hline
\end{tabular}
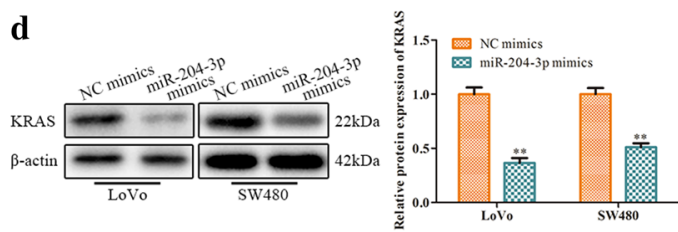

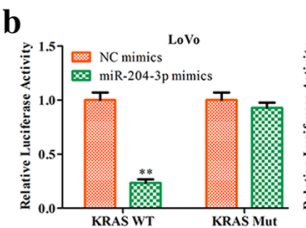

e

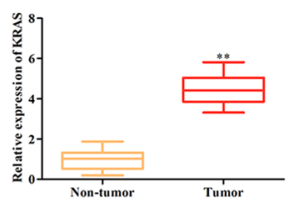

c

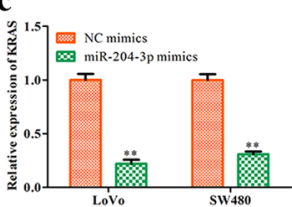

f

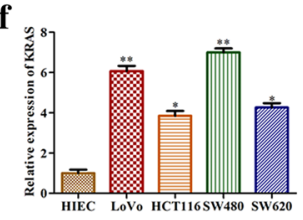

Fig. 6 KRAS is a target gene of miR-204-3p. a The predicted location of the KRAS combination according to TargetScan. $\mathbf{b}$ The results of the dual luciferase reporter assay. $\mathbf{c}, \mathbf{d}$ The effects of miR-204-3p overexpression on KRAS mRNA and protein expression in LoVo and SW480 cells. e KRAS mRNA levels in tumour tissues and HC tissues. Data from $15 \mathrm{HCs}$ and $15 \mathrm{GC}$ patients. $\mathbf{f}$ KRAS mRNA levels in HIECs and CRC cell lines. Values are the mean $\pm S D$. ${ }^{*} P<0.05,{ }^{* *} P<0.01$, and ${ }^{* *} P<0.001$ vs. NC mimics group, HC group or HIECs group. $\mathrm{n}=3$ per group
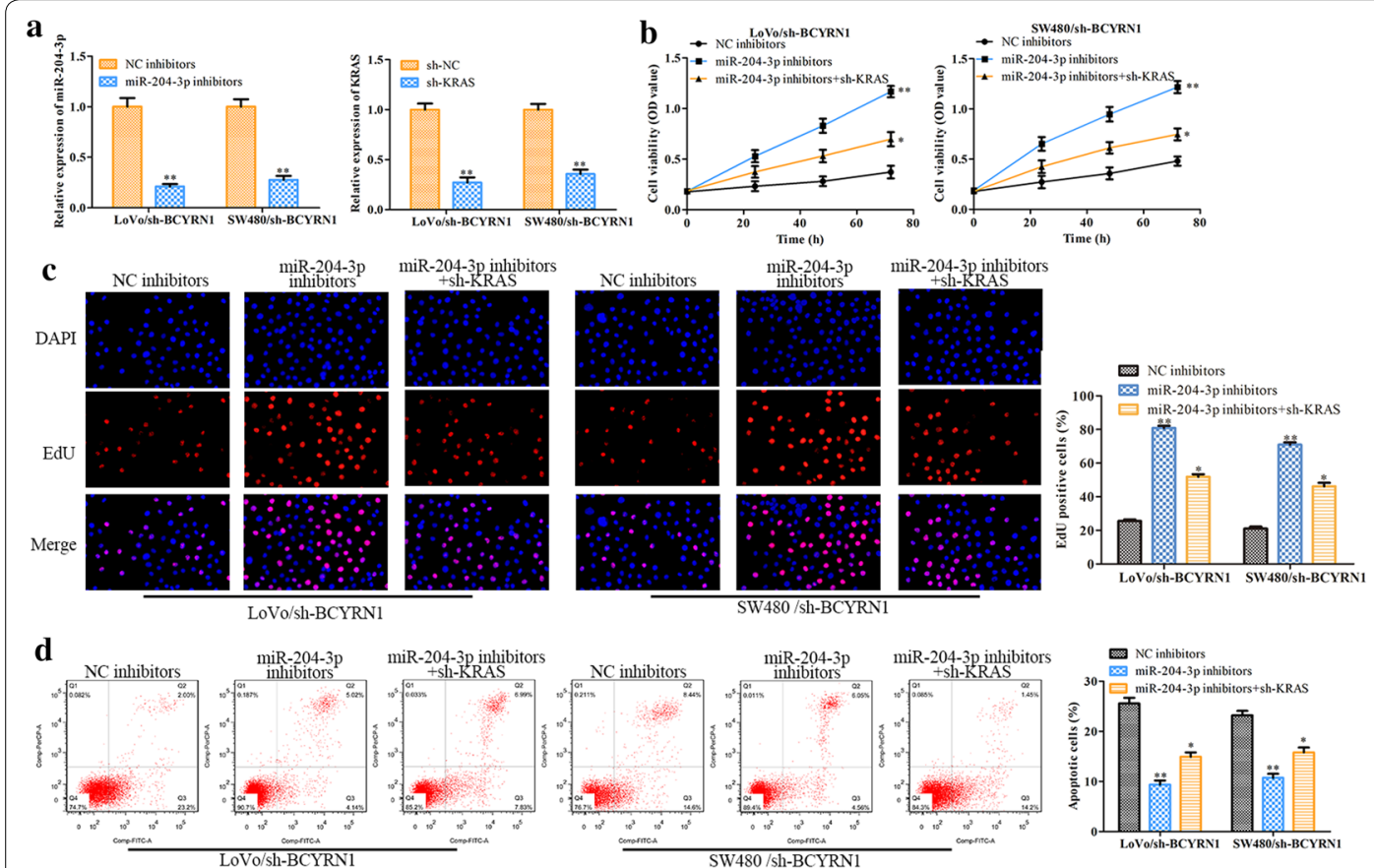

$\mathbf{e}$ $m i R-204-3 p$
inhibitors miR-204-3pinhibitors $\quad$ NC inhibitors $\underset{\text { inhibitors }}{\min -204-3 p} \begin{gathered}\text { miR-204-3p inhibitors } \\ \text { +sh-KRAS }\end{gathered}$
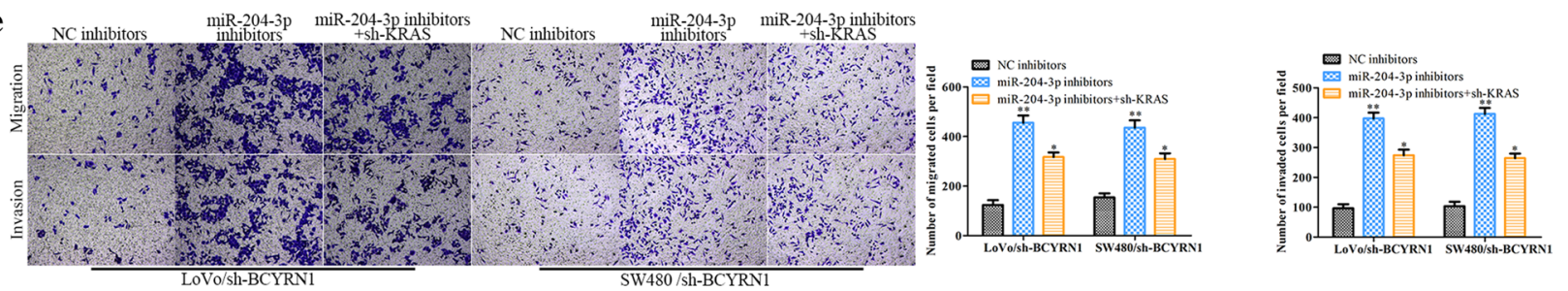

Fig. 7 The effects of the BCYRN1/miR-204-3p/KRAS axis on CRC progression in vitro. a miR-204-3p expression in LoVo and SW480 cells with BCYRN1 knockdown. b, c The effects of proliferation determined by CCK-8 assay and EdU analysis. d Original images and histogram of the flow cytometry assay. e Cell migration detected by Transwell assay. Values are the mean \pm SD. ${ }^{*} P<0.05$, ${ }^{* *} P<0.01$, and ${ }^{* * *} P<0.001$ vs. NC inhibitor group. ${ }^{*} P<0.05$, ${ }^{\# \#} P<0.01$, and ${ }^{\# \#} P<0.001$ vs. the miR-204-3p inhibitor group. $n=6$ per group 


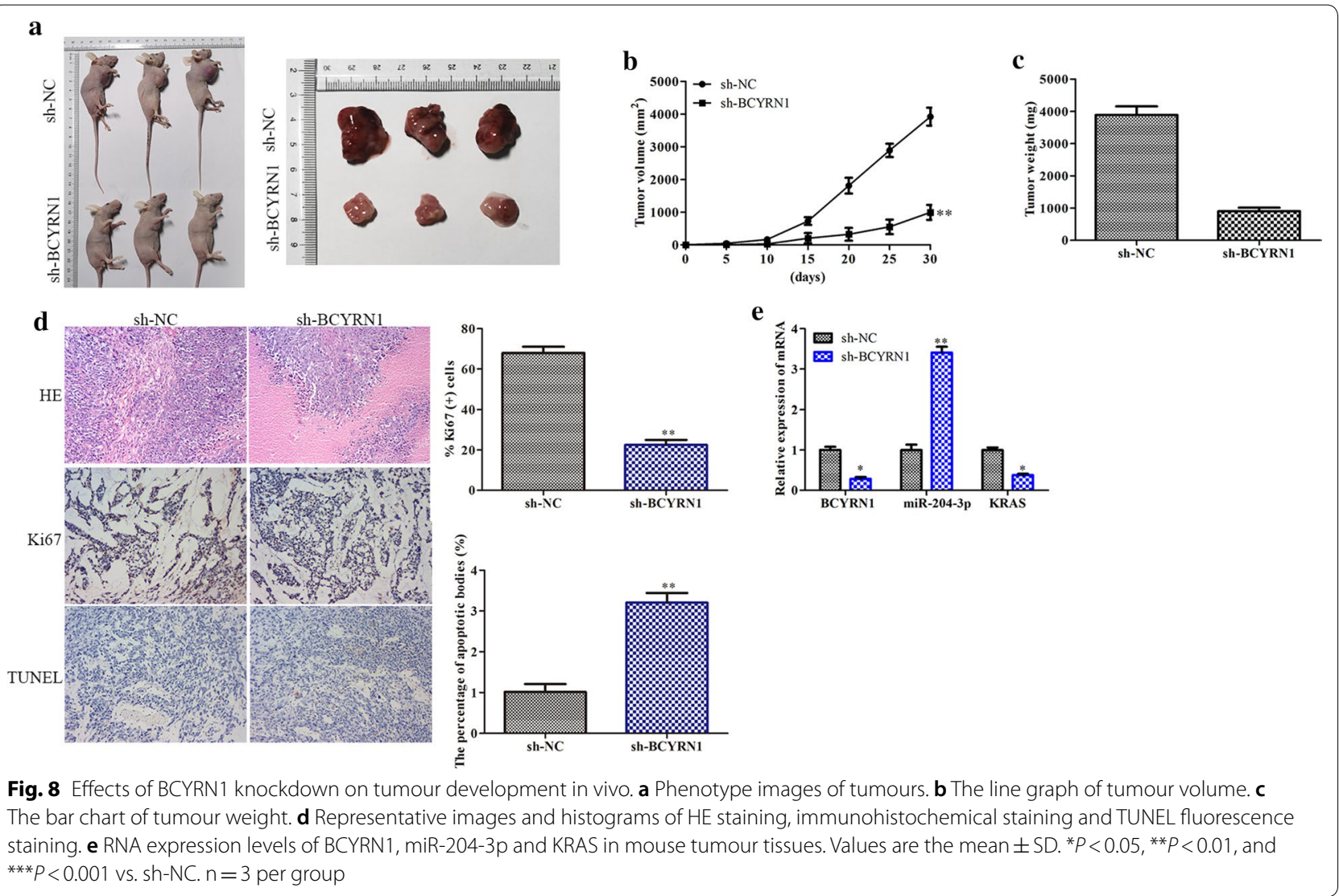

As mentioned above, miRNAs are involved in the regulation of cancer [22, 23]. Interestingly, miRNAs can act as tumour suppressor genes [44] or oncogenes [45] to regulate the biological characteristics of cancer. Cui et al. reported that miR-204-3p acts on its potential target gene fibronectin 1 (FN1) and inhibits its expression, thereby decreasing the growth of hepatocellular carcinoma tumour endothelial cells [46]. Recently, several studies have reported the roles of miR-204-3p in ovarian cancer [47], lung adenocarcinoma [48] and bladder cancer [49], but the functions of miR-204-3p in CRC remain obscure. Our current research showed that miR-204-3p is a potential target gene of BCYRN1 and is negatively regulated by BCYRN1. We further investigated whether miR-204-3p overexpression inhibited CRC cell proliferation, migration and invasion while exacerbating CRC cell apoptosis. In addition, bioinformatics analysis and dual luciferase reporter assay proved that KRAS was a potential target gene of miR-204-3p. KRAS is an important oncogene and a key tumour maintenance gene in many carcinomas [34]. Then, we showed that KRAS was highly expressed in CRC tissues and cell lines, and overexpression of miR204-3p eliminated this effect. These results indicated that overexpression of miR-204-3p and downregulation of
KRAS can be considered new approaches to the treatment of CRC.

In summary, these findings demonstrated that BCYRN1 participates in the occurrence and development of CRC. In terms of functional regulation, BCYRN1 promotes CRC progression at least in part by regulating the miR-204-3p/KRAS axis. Our study broadens the current understanding of BCYRN1 function in CRC and provides a novel therapeutic target for CRC.

\section{Conclusion}

1. BCYRN1 is highly expressed in CRC tissues and cell lines compared to normal controls.

2. Downregulation of BCYRN1 decreases the migration and invasion of CRC cells. Knockdown of BCYRN1 promotes the apoptosis of CRC cells.

3. miR-204-3p is a target gene of BCYRN1 and affects CRC cell proliferation, migration, invasion and apoptosis.

4. KRAS is a target gene of miR-204-3p, and the BCYRN1/miR-204-3p/KRAS axis affects the proliferation, migration, invasion and apoptosis of CRC cells.

5. Knockdown of BCYRN1 inhibits tumour growth. 


\section{Acknowledgements \\ None.}

\section{Authors' contributions}

Liu Yang conceived and designed the study. Liu Yang, Yinan Zhang, Jun Bao, and Ji-Feng Feng performed the literature search and data extraction. Liu Yang analysed the data and the manuscript. All authors read and approved the final manuscript.

\section{Funding}

This study was funded by the National Natural Science Foundation of China (81602145 to LY), Jiangsu Provincial Natural Science Foundation (BK20171509 to LY), Jiangsu Provincial Medical Youth Talent, The Project of Invigorating Health Care through Science, Technology Education (QNRC2016649 to LY), the China Postdoctoral Science Foundation (2018M632265 to LY), the Talents Program of Jiangsu Cancer Hospital (YC201812 to LY), and the "333 Talents" Program of Jiangsu Province (BRA2020390 to LY).

\section{Availability of data and materials}

Yes.

\section{Ethics approval and consent to participate}

This study was carried out based on the Ethics Committee of The Affiliated Cancer Hospital of Nanjing Medical University. The clinical analysis was performed according to the principles of the Helsinki Declaration.

\section{Consent for publication}

All authors agree to publish.

\section{Competing interests}

The authors declare that they have no competing interests.

\section{Author details}

${ }^{1}$ Department of Colorectal Surgery, The Affiliated Cancer Hospital of Nanjing Medical University \& Jiangsu Cancer Hospital \& Jiangsu Institute of Cancer Research, Nanjing, China. ${ }^{2}$ Department of Chemotherapy, The Affiliated Cancer Hospital of Nanjing Medical University \& Jiangsu Cancer Hospital \& Jiangsu Institute of Cancer Research, No. 42 Baiziting, Nanjing, China.

Received: 4 June 2020 Accepted: 5 September 2020

Published online: 14 September 2020

\section{References}

1. Bray F, Ferlay J, Soerjomataram I, et al. Global cancer statistics 2018: GLOBOCAN estimates of incidence and mortality worldwide for 36 cancers in 185 countries. CA Cancer J Clin. 2018;68(6):394-424.

2. Arnold M, Sierra MS, Laversanne M, et al. Global patterns and trends in colorectal cancer incidence and mortality. Gut. 2017;66(4):683-91.

3. Brenner $\mathrm{H}$, Kloor M, Pox CP. Colorectal cancer. Lancet. 2014;383(9927):1490-502

4. Dekker E, Rex DK. Advances in CRC prevention: screening and surveillance. Gastroenterology. 2018;154(7):1970-84.

5. Schreuders EH, Ruco A, Rabeneck L, et al. Colorectal cancer screening: a global overview of existing programmes. Gut. 2015;64(10):1637-49.

6. Neureiter $D$, Jäger $T$, Ocker $M$, et al. Epigenetics and pancreatic cancer: pathophysiology and novel treatment aspects. World J Gastroenterol. 2014:20:7830-48.

7. Michalak EM, Burr ML, Bannister AJ, et al. The roles of DNA, RNA and histone methylation in ageing and cancer. Nat Rev Mol Cell Biol. 2019;20(10):573-89.

8. Cech TR, Steitz JA. The noncoding RNA revolution-trashing old rules to forge new ones. Cell. 2014;157:77-94.

9. Tay Y, Rinn J, Pandolfi PP. The multilayered complexity of ceRNA crosstalk and competition. Nature. 2014:505:344-52.

10. Marchese FP, Huarte M. Long non-coding RNAs and chromatin modifiers: their place in the epigenetic code. Epigenetics. 2014;9(1):21-6.

11. Thomson DW, Dinger ME. Endogenous microRNA sponges: evidence and controversy. Nat Rev Genet. 2016;17(5):272-83.
12. Wang $P$, Xue $Y$, Han Y, et al. The STAT3-binding long noncoding RNA Inc-DC controls human dendritic cell differentiation. Science. 2014:344(6181):310-3.

13. Prensner JR, lyer MK, Sahu A, et al. The long noncoding RNA SChLAP1 promotes aggressive prostate cancer and antagonizes the SWI/SNF complex. Nat Genet. 2013;45:1392-8.

14. Hung $C L$, Wang $L Y, Y u Y L$, et al. A long noncoding RNA connects c-Myc to tumor metabolism. Proc Natl Acad Sci USA. 2014;111(52):18697-702.

15. Hu T, LuY. BCYRN1, a c-MYC-activated long non-coding RNA, regulates cell metastasis of non-small-cell lung cancer. Cancer Cell Int. 2015;15:36.

16. Booy EP, McRae EK, Koul A, et al. The long non-coding RNA BC200 (BCYRN1) is critical for cancer cell survival and proliferation. Mol Cancer. 2017;16(1):109.

17. Ding $S$, Jin $Y$, Hao $Q$, et al. LncRNA BCYRN1/miR-490-3p/POU3F2, served as a ceRNA network, is connected with worse survival rate of hepatocellular carcinoma patients and promotes tumor cell growth and metastasis. Cancer Cell Int. 2020;20:6

18. Zhai H, Li Y. BCYRN1 is correlated with progression and prognosis in gastric cancer. Biosci Rep. 2019. https://doi.org/10.1042/BSR20190505.

19. Wang YC, Chuang YH, Shao Q, et al. Brain cytoplasmic RNA 1 suppresses smooth muscle differentiation and vascular development in mice. J Biol Chem. 2018;293(15):5668-78

20. Zhang $X Y$, Tang $X Y$, Ma LJ, et al. Schisandrin B down-regulated IncRNA BCYRN1 expression of airway smooth muscle cells by improving miR-150 expression to inhibit the proliferation and migration of ASMC in asthmatic rats. Cell Prolif. 2017;50(6):e12382.

21. Ghildiyal M, Zamore PD. Small silencing RNAs: an expanding universe. Nat Genet. 2009;10:94-108

22. Ambros V. The functions of animal microRNAs. Nature 2004:431:350-5.

23. Bartel DP. MicroRNAs: genomics, biogenesis, mechanism, and function. Cell. 2004;116:281-97.

24. Lim LP, Lau NC, Garrett-Engele P, et al. Microarray analysis shows that some microRNAs downregulate large numbers of target mRNAs. Nature. 2005:433:769-73.

25. Ren XS, Tong Y, Qiu Y, et al. MiR155-5p in adventitial fibroblasts-derived extracellular vesicles inhibits vascular smooth muscle cell proliferation via suppressing angiotensin-converting enzyme expression. J Extracell Vesicles. 2019:9(1):1698795

26. Necsulea A, Soumillon M, Warnefors $M$, et al. The evolution of IncRNA repertoires and expression patterns in tetrapods. Nature. 2014;505(7485):635-40.

27. Qu L, Ding J, Chen C, et al. Exosome-transmitted IncARSR promotes sunitinib resiistance in renal cancer by acting as a competing endogenous RNA. Cancer Cell. 2016:29(5):653-68.

28. Gates EDH, Lin JS, Weinberg JS, et al. Guiding the first biopsy in glioma patients using estimated Ki-67 maps derived from MRI: conventional versus advanced imaging. Neuro-Oncology. 2019;21(4):527-36.

29. Pan Z, Cai J, Lin J, et al. A novel protein encoded by circFNDC3B inhibits tumor progression and EMT through regulating Snail in colon cancer. Mol Cancer. 2020. https://doi.org/10.1186/s12943-020-01179-5.

30. Nagaraju GP, El-Rayes BF. Cyclooxygenase-2 in gastrointestinal malignancies. Cancer. 2019;125(8):1221-7.

31. La Rocca G, Pucci-Minafra I, Marrazzo A, et al. Zymographic detection and clinical correlations of MMP-2 and MMP-9 in breast cancer sera. Br J Cancer. 2004;90(7):1414-21.

32. Hu L, Chen M, Chen X, et al. Chemotherapy-induced pyroptosis is mediated by BAK/BAX-caspase-3-GSDME pathway and inhibited by 2-bromopalmitate. Cell Death Dis. 2020;11(4):281.

33. Walensky LD. Targeting BAX to drug death directly. Nat Chem Biol. 2019:15:657-65.

34. Terhune PG, Phifer DM, Tosteson TD, et al. K-ras mutations in focal prolif eration lesions of human pancreas. Cancer Epidemiol Biomarkers Prev. 1998;7(6):515-21

35. Siegel RL, Miller KD, Jemal A, et al. Cancer statistics. CA Cancer J Clin. 2017:67:7-30.

36. Punt CJA, Koopman M, Vermeulen $L$, et al. From tumour heterogeneity to advances in precision treatment of colorectal cancer. Nat Rev Clin Oncol. 2017;14:235-46.

37. Kuipers EJ, Grady WM, Lieberman D, et al. Colorectal cancer. Nat Rev Dis Primers. 2015;1:15065. 
38. Yang L, Lin C, Jin C, et al. IncRNA-dependent mechanisms of androgenreceptor-regulated gene activation programs. Nature. 2013:500:598-602.

39. Lorenzen J, Thum T. Long noncoding RNAs in kidney and cardiovascular diseases. Nat Rev Nephrol. 2016;12:360-73.

40. Yu W, Xiang $D$, Jia $H$, et al. The IncRNA BCYRN1 functions as an oncogene in human glioma by downregulating miR-125a-5p in vitro. Cancer Manag Res. 2020;12:1151-61.

41. Lang N, Wang C, Zhao J, et al. Long non-coding RNA BCYRN1 promotes glycolysis and tumor progression by regulating the miR-149/PKM2 axis in non-small-cell lung cancer. Mol Med Rep. 2020;21(3):1509-16.

42. Gu L, Lu L, Zhou D, et al. Long noncoding RNA BCYRN1 promotes the proliferation of colorectal cancer cells via up-regulating NPR3 expression. Cell Physiol Biochem. 2018:48(6):2337-499.

43. Yu JH, Chen Y. Clinical significance of IncRNA BCYRN1 in colorectal cancer and its role in cell metastasis. Eur Rev Med Pharmacol Sci. 2019;23(21):9371-8.

44. Suzuki HI, Miyazono K. Emerging complexity of microRNA generation cascades. J Biochem. 2011;149:15-25.

45. Newman MA, Hammond SM. Emerging paradigms of regulated microRNA processing. Genes Dev. 2010;24:1086-92.
46. Cui ZH, Shen SQ, Chen ZB, et al. Growth inhibition of hepatocellular carcinoma tumor endothelial cells by miR-204-3p and underlying mechanism World J Gastroenterol. 2014;20(18):5493-504.

47. Yuan D, Qian H, Guo T, et al. LncRNA-ATB promotes the tumorigenesis of ovarian cancer via targeting miR-204-3p. Onco Targets Ther. 2020;13:573-83.

48. Yang S, Liu T, Sun Y, et al. The long noncoding RNA LINC00483 promotes lung adenocarcinoma progression by sponging miR-204-3p. Cell Mol Biol Lett. 2019;24:70.

49. Guo J, Zhao P, Liu Z, et al. MiR-2014-3p inhibited the proliferation of bladder cancer cells via modulating lactate dehydrogenase-mediated glycolysis. Front Oncol. 2019;9:1242.

\section{Publisher's Note}

Springer Nature remains neutral with regard to jurisdictional claims in published maps and institutional affiliations.
Ready to submit your research? Choose BMC and benefit from:

- fast, convenient online submission

- thorough peer review by experienced researchers in your field

- rapid publication on acceptance

- support for research data, including large and complex data types

- gold Open Access which fosters wider collaboration and increased citations

- maximum visibility for your research: over $100 \mathrm{M}$ website views per year

At BMC, research is always in progress.

Learn more biomedcentral.com/submissions 